\title{
Substance use disorders and co-morbidities among Asian Americans and Native Hawaiians/Pacific Islanders
}

\author{
L.-T. $W u^{1,2 *}$ and D. G. Blazer ${ }^{1 *}$ \\ ${ }^{1}$ Department of Psychiatry and Behavioral Sciences, School of Medicine, Duke University Medical Center, Durham, NC, USA \\ ${ }^{2}$ Center for Child and Family Policy, Duke University, Durham, NC, USA
}

Background. Asian Americans (AAs) and Native Hawaiians/Pacific Islanders (NHs/PIs) are the fastest growing segments of the US population. However, their population sizes are small, and thus AAs and NHs/PIs are often aggregated into a single racial/ethnic group or omitted from research and health statistics. The groups' substance use disorders (SUDs) and treatment needs have been under-recognized.

Method. We examined recent epidemiological data on the extent of alcohol and drug use disorders and the use of treatment services by AAs and NHs/PIs.

Results. NHs/PIs on average were less educated and had lower levels of household income than AAs. Considered as a single group, AAs and NHs/PIs showed a low prevalence of substance use and disorders. Analyses of survey data that compared AAs and NHs/PIs revealed higher prevalences of substance use (alcohol, drugs), depression and delinquency among NHs than among AAs. Among treatment-seeking patients in mental healthcare settings, NHs/PIs had higher prevalences of DSM-IV diagnoses than AAs (alcohol/drug, mood, adjustment, childhood-onset disruptive or impulsecontrol disorders), although co-morbidity was common in both groups. AAs and NHs/PIs with an SUD were unlikely to use treatment, especially treatment for alcohol problems, and treatment use tended to be related to involvement with the criminal justice system.

Conclusions. Although available data are limited by small sample sizes of AAs and NHs/PIs, they demonstrate the need to separate AAs and NHs/PIs in health statistics and increase research into substance use and treatment needs for these fast-growing but understudied population groups.

Received 22 January 2014; Revised 12 May 2014; Accepted 16 May 2014; First published online 20 June 2014

Key words: Addiction treatment, Asian Americans, co-morbidity, Native Hawaiians, Pacific Islanders, substance use disorder.

\section{Introduction}

Asian Americans (AAs) include people with origins in the Far East, Southeast Asia and the Indian subcontinent (Asian Indians, Chinese, Filipinos, Japanese, Koreans, Vietnamese, and other Asians). Native Hawaiians and other Pacific Islanders (NHs/PIs) include people having origins in Hawaii, Guam, Samoa, or other Pacific Islands. Data from the 2010 US Census showed that AAs and NHs/PIs were the fastest growing segments of the US population (US Census Bureau, 2011), increasing at least three times faster than the total US population. In 2011, an

* Author for correspondence: Dr L.-T. Wu, Department of Psychiatry and Behavioral Sciences, School of Medicine, Duke University Medical Center, Durham, NC, USA.

(Email: litzy.wu@duke.edu) [L.-T.W.]

(Email: dan.g.blazer@dm.duke.edu) [D.G.B.] estimated 18.2 million AAs and 1.4 million NHs/PIs were US residents (US Census Bureau, 2013).

Substance use disorders (SUDs) and treatment needs among AAs and NHs/PIs have been understudied in comparison with other racial/ethnic groups. In the 1990 census, self-designated AAs and NHs/PIs were classified as one group. A 1997 directive from the Office of Management and Budget called for separating AAs and NHs/PIs in census reporting, and in 2000 the census began reporting the groups separately. Epidemiological and clinical studies of substance use and SUDs have often combined the two groups as 'Asian Americans and Pacific Islanders (AAPI)' or reported the pooled groups as simply 'Asians', which is not optimal for a variety of reasons. At least $20 \mathrm{sub}-$ groups make up the 17 million people who designate themselves as AAs and NHs/PIs (SAMHSA, 2010a). The statistical power in almost all data sets used to estimate prevalence and correlates of SUDs is not sufficient to disaggregate the individual groups. 
Table 1. Demographics of Asian Americans and Native Hawaiians/Pacific Islanders in the USA

\begin{tabular}{|c|c|c|}
\hline & Asian Americans & Native Hawaiians/Pacific Islanders \\
\hline Population size, 2000 census & 11.9 million & 874000 \\
\hline \multirow[t]{2}{*}{ Population size, 2010 census } & 17.3 million & 1.2 million \\
\hline & $5.6 \%$ of the US population & $0.4 \%$ of the US population \\
\hline $\begin{array}{l}\text { Percentage growth: } \\
2000 \text { v. } 2010\end{array}$ & $46 \%$ & $40 \%$ \\
\hline $\begin{array}{l}\text { Percentage reporting } \\
\text { multiple race }\end{array}$ & $15.3 \%$ & $55.9 \%$ \\
\hline Geographic distribution & $\begin{array}{l}\text { West } 46 \% \text {, South } 22 \% \text {, Northeast } 20 \% \text {, } \\
\text { Midwest } 12 \%\end{array}$ & $\begin{array}{l}\text { West } 71 \% \text {, South } 16 \% \text {, Northeast } 7 \% \text {, } \\
\text { Midwest } 6 \%\end{array}$ \\
\hline $\begin{array}{l}\text { States with relatively high } \\
\text { numbers of the population }\end{array}$ & $\begin{array}{l}\text { California ( } 5.6 \text { million), New York (1.6 million), } \\
\text { Texas (1.1 million), New Jersey (0.8 million), } \\
\text { Hawaii ( } 0.8 \text { million), Illinois ( } 0.7 \text { million), } \\
\text { Washington ( } 0.6 \text { million), Florida ( } 0.6 \text { million), } \\
\text { Virginia (0.5 million), Pennsylvania ( } 0.4 \text { million) }\end{array}$ & $\begin{array}{l}\text { Hawaii (356000), California ( } 286000) \text {, } \\
\text { Washington (70000), Texas ( } 48000) \text {, Florida } \\
\text { (40000), Utah (37000), New York (36000), } \\
\text { Nevada (33000), Oregon (26000), Arizona } \\
\text { (25000). }\end{array}$ \\
\hline Larger subgroups & $\begin{array}{l}\text { Chinese (4 million), Filipinos ( } 3.4 \text { million), Asian } \\
\text { Indians ( } 3.2 \text { million), Vietnamese (1.7 million), } \\
\text { Koreans (1.7 million), Japanese (1.3 million) }\end{array}$ & $\begin{array}{l}\text { Native Hawaiian (527077), Samoan (184440), } \\
\text { Guamanian or Chamorro (147798) }\end{array}$ \\
\hline Poverty rate & $12.8 \%$ (for the Asian alone population in 2011 ) & $\begin{array}{l}21.5 \% \text { (for the Native Hawaiians and Other } \\
\text { Pacific Islanders alone population in 2011) }\end{array}$ \\
\hline Education & $\begin{array}{l}50 \% \text { (for the Asian alone population aged } \\
\geqslant 25 \text { years who had a bachelor's degree or } \\
\text { higher level of education in 2011) }\end{array}$ & $\begin{array}{l}14.5 \% \text { (for the Native Hawaiians and Other } \\
\text { Pacific Islanders alone aged } \geqslant 25 \text { years who } \\
\text { had a bachelor's degree or higher in 2011) }\end{array}$ \\
\hline
\end{tabular}

Sources of data: US census $(2012 a, b, 2013)$.

The acculturation and enculturation experiences of AAs and NHs/PIs in the dominant Caucasian culture have been vastly different. AAs have arrived in the USA as immigrants (e.g. the arrival of the first Japanese immigrants in 1843 and the role of Chinese workers in building the transcontinental railroad during 1865-1869), and even today, the majority of AAs are foreign-born (SAMHSA, 2010a). By contrast, $\mathrm{NHs} / \mathrm{PIs}$ are natives of their homelands, which have been annexed by the USA and populated by dominant American racial/ethnic groups. Many NHs/PIs move away from their native homes to other geographic locations in the USA. These differences in background between the groups may contribute to different substance use behaviors.

In response to the growing sizes of AAs and $\mathrm{NHs} /$ PIs and the need for empirical data to inform research efforts and health policy, we reviewed population and clinical data about substance use, SUDs (alcohol and drugs) and SUD treatment use by these two groups. To provide the context for the review, we described national data on demographics of AAs and NHs/PIs, compared prevalences of alcohol and drug use, and summarized estimates of SUDs. Finally, we considered prevalences of use of alcohol or drug abuse services and psychiatric co-morbidities as they related to treatment needs for AAs and NHs/PIs.

\section{Method}

We searched PubMed and Google Scholar using the key words Asian Americans, Native Hawaiians, Pacific Islanders, substance use, alcohol use, marijuana use, drug use, SUD (alcohol, drugs), substance abuse treatment, and co-morbidity. We excluded case reports, non-empirical (data-based) reports, and publications not in English. We used the related citations link to find additional publications. To reflect recent trends in substance use among AAs and NHs/PIs, we focused on the findings from population-based studies in the past 5 years that had an adequate sample size.

\section{Demographics of Asian Americans versus Native Hawaiians/Pacific Islanders}

AAs are the fastest growing racial/ethnic group in the country (see Table 1). By 2050, AAs are projected to make up about $9 \%$ (40.6 million) of the US population (US Census, 2011b). Two-thirds of AAs are foreignborn; $57 \%$ of these were naturalized US citizens in 2010. Forty-two percent speak English well, yet only $11 \%$ speak only English. The vast majority $(76 \%)$ of AAs live in large metropolitan areas ( $\mathrm{Wu}$ et al. 2013b). One half of the AA-alone population aged $\geqslant 25$ years have a bachelor's degree or higher level of education (US Census, 2013). AAs resemble Whites 
in having higher levels of family income than other racial/ethnic groups (DeNavas-Walt et al. 2012; Wu et al. 2014).

NHs/PIs (1.4 million in 2011) are growing in numbers at 3-4 times the rate of the overall US population (US Census, 2012b, 2013). States with relatively high proportions of NHs/PIs are Hawaii, Alaska, Utah, Nevada and Washington (US Census, 2012a). Just over half of NHs/PIs live in large metropolitan areas (Wu et al. 2013b). The NH/PI population (median age: 27.1 years) is younger than the AA population (median age: 33.5 years) and the overall US population (median age: 37.3 years) (US Census, 2013). About 30\% speak a language other than English at home (HHS, 2013). In 2011, $14.5 \%$ of NHs/PIs aged $\geqslant 25$ years had a bachelor's degree or higher level of education (US Census, 2013). In summary, NHs/PIs are less educated and less likely to be foreign-born compared to AAs, and they are more likely to speak English as their only language.

\section{Alcohol and drug use}

Because studies of SUDs typically include a small sample size of AAs and NHs/PIs, research has focused mainly on descriptive prevalences of alcohol and any drug use (see Table 2). AAs and NHs/PIs have either been pooled into an 'AAPI' group or combined with other racial/ethnic groups as 'other'. There is limited information about prevalences of use of illicit drugs among AAs versus NHs/PIs (Wu et al. 2013b,c). Earlier studies of AAs show that those who were born in the USA and AAs of mixed heritage had elevated prevalences of alcohol/drug use (Price et al. 2002; Wong et al. 2004).

A 2010 report used data from the 2004-2008 National Surveys on Drug Use and Health (NSDUH) to present findings on alcohol and drug use prevalences among AAs aged $\geqslant 18$ years (SAMHSA, 2010a). AAs had lower past-month prevalences of alcohol and drug use than the national average: alcohol use $(39.8 \%$ v. $55.2 \%)$, binge alcohol use $(13.2 \%$ v. $24.5 \%)$ and illicit/non-medical drug use (3.4\% v. $7.9 \%)$. Past-month alcohol use was reported by $51.9 \%$ of Korean Americans, $48.3 \%$ of Japanese Americans, $41.3 \%$ of Chinese Americans, 38.7\% of Vietnamese Americans, 38.1\% of Filipino Americans and 32.1\% of Asian Indians. Binge drinking varied from 25.9\% among Korean Americans to $8.4 \%$ among Chinese Americans (Filipino Americans 15.0\%, Japanese Americans 14.5\%, Vietnamese Americans 14.0\%, and Asian Indians 9.5\%). The prevalence of any illicit or non-medical drug use also varied (Korean Americans 5.3\%, Japanese Americans 6.2\%, Chinese Americans
2.1\%, Vietnamese Americans 5.3\%, Filipino Americans $3.2 \%$, and Asian Indians 2.1\%).

A similar pattern is noted for substance use among adolescents (12-17 years) from the pooled 2004-2009 NSDUH data (SAMHSA, 2011), as shown in Table 2. Prevalence of alcohol use ranged from $9.7 \%$ among Filipino Americans to $5.1 \%$ among Asian Indians $5.1 \%$. Marijuana use varied from 5.2\% among Korean Americans to $1.0 \%$ among Asian Indians. Available drug use estimates for NHs/PIs are even more limited, as studies have often pooled NHs/PIs with other racial/ ethnic groups. Studies that separated AAs and NHs/ PIs in the analysis have demonstrated differences in behavioral and mental health between AAs and NHs/PIs (Wong et al. 2004; Kim \& McCarthy, 2006; Wu et al. 2013a,b,c). For example, Lowry et al. (2011) pooled data from 4 years (2001, 2003, 2005, and 2007) of the Youth Risk Behavior Survey to examine behavioral health among high school students $(n=56773)$. $\mathrm{NHs} / \mathrm{PIs}$ had higher prevalences than AAs in current substance use (see Table 2), and also in being sexually active, carrying a weapon, engaging in a physical flight, suicidal ideation, and attempting suicide. Higher prevalences of these behavioral health indicators were observed in both genders of NHs/PIs and showed little gender differences. Overall, AAs showed lower prevalences, and NHs/PIs were similar to Whites, Blacks and Hispanics in prevalence of these behavioral health indicators (Lowry et al. 2011).

Recently, Wu et al. (2013b) examined national trends in illicit/non-medical drug use prevalences among AAs and NHs/PIs aged $\geqslant 12$ years for 2005-2011 $(n=275$ 685) (see Table 2). Between 2005 and 2011, there were no significant variations in past-year prevalences of drug use among NHs/PIs and AAs. Drug use was much more common among NHs/PIs than AAs and Whites. Controlling for multiple covariates, NHs/PIs showed higher adjusted odds ratios (aORs) than AAs for any drug use [aOR 1.70, 95\% confidence interval (CI) 1.20-2.40], marijuana use (aOR 1.72, 95\% CI 1.12-2.63) and heroin use (aOR 5.06, 95\% CI 1.2520.49). Adjusted analyses also found that NHs/PIs resembled Whites in odds of use of most drugs.

Regardless of race/ethnicity, age was the most reliable correlate of recent illicit or non-medical drug use among AAs and NHs/PIs, which generally showed an increase in adolescence and young adulthood, then a decline thereafter (Wu et al. 2013b, 2014). Low household income was associated with elevated odds of drug use among AAs but not among NHs/PIs; other demographics (e.g. gender, urbanicity, residential move) were inconsistent correlates of drug use. However, behavioral and mental health variables (e.g. delinquency, being arrested, tobacco use, alcohol use, major depression) were associated with past-year 
Table 2. Prevalences of alcohol and illicit/non-medical drug use among Asian Americans (AAs) and Native Hawaiians/Pacific Islanders (NHs/PIs)

\begin{tabular}{|c|c|c|c|c|}
\hline Authors/citation & Dataset, Age & Sample size & Substance & Selected findings \\
\hline SAMHSA, 2011 & $\begin{array}{l}\text { National sample } \\
\text { NSDUH } \\
12-17 \text { years }\end{array}$ & 3763 AAs & Alcohol and drugs & $\begin{array}{l}\text { AA past-month use prevalence } \\
\text { Alcohol } 7.4 \% \text {, marijuana } 2.9 \% \text {, prescription drugs } 1.8 \% \\
\text { Born in the USA } \\
\text { Alcohol } 8.7 \% \text {, marijuana } 3.2 \% \text {, prescription drugs } 1.4 \% \\
\text { Not born in the USA } \\
\text { Alcohol } 4.7 \% \text {, marijuana } 2.1 \% \text {, prescription drugs } 2.7 \% \\
\text { Aged } 12-14 \text { years } \\
\text { Alcohol } 2.7 \% \text {, marijuana } 0.5 \% \text {, prescription drugs } 1.6 \% \\
\text { Aged } 15-17 \text { years } \\
\text { Alcohol } 11.5 \% \text {, marijuana } 4.9 \% \text {, prescription drugs } 2.0 \%\end{array}$ \\
\hline Lowry et al. 2011 & $\begin{array}{l}\text { National sample } \\
\text { YRBSS } \\
\text { High school students, } \\
\text { age unavailable }\end{array}$ & 56773 & Alcohol and drugs & $\begin{array}{l}\text { Past-month use prevalence } \\
\text { AA: alcohol } 25.6 \% \text {, heavy drinking } 12.1 \% \text {, marijuana } 9.0 \% \\
\text { NH/PI: alcohol } 44.2 \% \text {, heavy drinking } 32.3 \% \text {, marijuana } 23.0 \% \\
\text { Female AA: alcohol } 24.9 \% \text {, heavy drinking } 10.2 \% \text {, marijuana } 9.2 \% \\
\text { Male AA: alcohol } 26.2 \% \text {, heavy drinking } 13.8 \% \text {, marijuana } 8.8 \% \\
\text { Female NH/PI: alcohol } 45.7 \% \text {, heavy drinking } 31.5 \% \text {, marijuana } 19.5 \% \\
\text { Male NH/PI: alcohol } 42.5 \% \text {, heavy drinking } 33.2 \% \text {, marijuana } 26.8 \%\end{array}$ \\
\hline SAMHSA, $2010 a$ & $\begin{array}{l}\text { National sample } \\
\text { NSDUH } \\
\geqslant 18 \text { years }\end{array}$ & 7629 AAs & Alcohol and drugs & $\begin{array}{l}\text { AA past-month use prevalence } \\
\text { Alcohol } 39.8 \% \text {, binge alcohol } 13.2 \% \text {, illicit or non-medical drugs } 3.4 \% \\
\text { Born in the USA } \\
\text { Alcohol } 56.0 \% \text {, binge alcohol } 22.0 \% \text {, illicit or non-medical drugs } 7.3 \% \\
\text { Not born in the USA } \\
\text { Alcohol } 35.9 \% \text {, binge alcohol } 11.1 \% \text {, illicit or non-medical drugs } 2.5 \% \\
\text { Alcohol use by age } \\
\text { Female: } 18-25 \text { years } 43.6 \%, 26-49 \text { years } 32.0 \%, \geqslant 50 \text { years } 18.3 \% \\
\text { Male: } 18-25 \text { years } 53.5 \%, 26-49 \text { years } 53.6 \%, \geqslant 50 \text { years } 45.0 \% \\
\text { Illicit or non-medical drug use by age } \\
\text { Female: } 18-25 \text { years } 6.9 \%, 26-49 \text { years } 1.4 \%, \geqslant 50 \text { years } 3.5 \% \\
\text { Male: } 18-25 \text { years } 11.4 \%, 26-49 \text { years } 3.1 \%, \geqslant 50 \text { years } 1.0 \%\end{array}$ \\
\hline Wu et al. $2013 b$ & $\begin{array}{l}\text { National sample } \\
\text { NSDUH } \\
\geqslant 12 \text { years }\end{array}$ & 275685 & Drugs & $\begin{array}{l}\text { AA: past-year use prevalence in } 2011 \\
\text { Any drug } 6.8 \% \text {, marijuana } 4.9 \% \text {, opioid analgesic } 1.8 \% \text {, inhalant } 0.7 \% \text {, cocaine } 0.8 \% \text {, } \\
\text { hallucinogen } 1.0 \% \text {, heroin }<1.0 \% \text {, stimulant } 0.3 \% \text {, sedative }<1.0 \% \text {, tranquilizer } 0.8 \% \\
\text { NH/PI: past-year use prevalence in } 2011 \\
\text { Any drug } 21.2 \% \% \text {, marijuana } 18.8 \% \text {, opioid analgesic } 2.7 \% \text {, inhalant } 2.7 \% \text {, cocaine } 3.2 \% \text {, } \\
\text { hallucinogen } 3.8 \% \text {, heroin } 0.8 \% \text {, stimulant } 1.7 \% \text {, sedative }<1.0 \% \text {, tranquilizer } 1.5 \% \\
\text { Adjusted OR }(95 \% \text { CI) of past-year use } \\
\text { NH/PI } v \text {. AA: any drug use } 1.70(1.20-2.40) \text {, marijuana use } 1.72(1.12-2.63) \text {, heroin use } 5.06(1.25-20.49) \\
\text { NH/PI } v \text {. White: any drug use } 1.01(0.76-1.35) \text {, marijuana use } 0.87(0.60-1.27) \text {, heroin use } 1.63(0.67-3.99) \\
\text { AA } v \text {. White: any drug use } 0.60(0.52-0.68) \text {, marijuana use } 0.51(0.44-0.59) \text {, heroin use } 0.32(0.10-1.02)\end{array}$ \\
\hline
\end{tabular}


drug use among both AAs and NHs/PIs (Wu et al. 2013b, 2014).

\section{Alcohol and drug use disorders}

Data from the 2001-2003 National Comorbidity Survey Replication (NCS-R) showed that about $7.9 \%$ of White, $6.3 \%$ of Black and $9.1 \%$ of Hispanic adults aged $\geqslant 18$ years met criteria for having a past-year alcohol use disorder (Breslau et al. 2005). However, because of the small sample size, estimates for AAs and NHs/ PIs are not available. The 2001-2002 National Epidemiologic Survey on Alcohol and Related Conditions (NESARC) included a total of 1332 AA/NH/PI adults aged $\geqslant 18$ years. In the NESARC, the pooled AAs/ $\mathrm{NHs} / \mathrm{PIs}$ had a lower prevalence of past-year alcohol use disorder than other groups (Hasin et al. 2007), and there were slighter racial/ethnic differences in the prevalence of past-year drug use disorder (Compton et al. 2007), as shown in Table 3.

Among adolescents (age 12-17 years), analyses of data from the 2005-2008 NSDUH $(n=72561)$ showed that the pooled AA/NH/PI group was similar to Blacks in prevalence of past-year SUDs but had lower prevalences than Whites, Hispanics, Native Americans and mixed-race people (Wu et al. 2011). Conditional prevalences of alcohol or drug use disorders among alcohol or drug users (the liability to abuse or dependence given use) suggested that the pooled $\mathrm{AA} / \mathrm{NH} / \mathrm{PI}$ group was similar to Blacks in the prevalence of drug use disorder given use $(\mathrm{Wu}$ et al. 2011).

In an analysis of SUDs by emergency department use in national samples of adults aged $\geqslant 18$ years $(n=113672)$, pooled AAs/NHs/PIs also showed a comparatively low prevalence of SUDs given use (Wu et al. 2012). Among past-year alcohol or drug users who received care in an emergency department setting, $11.7 \%$ of pooled AAs/NHs/PIs, $15.2 \%$ of Whites, $18.0 \%$ of Blacks, $17.2 \%$ of Hispanics, $28.7 \%$ of Native Americans and $23.6 \%$ of mixed-race adults met criteria for having an SUD. In controlled analyses, AAs/NHs/ PIs did not differ from alcohol-using Whites in the odds of alcohol use disorders but drug-using AAs/ $\mathrm{NHs} / \mathrm{PIs}$ had lower odds of drug use disorders than drug-using Whites.

A more recent study of marijuana use disorder in a national sample of persons aged $\geqslant 12$ years separated NHs/PIs and AAs (Wu et al. 2014) (see Table 3). In the overall sample, more NHs/PIs than AAs used marijuana in the past year but there were no significant differences in past-year marijuana use disorder in either the total sample $(1.3 \% v .0 .8 \%)$ or the subsample of marijuana users $(12.6 \%$ v. $17.0 \%)$. Controlling for sociodemographic variables, AAs and Hispanics had lower odds of marijuana use than Whites, the odds for Blacks and Native Americans resembled those of Whites, and mixed-race persons had greater odds than Whites of using marijuana. Among marijuana users, AAs had greater odds than Whites of having a marijuana use disorder (aOR 1.88, 95\% CI 1.39-2.55), and NH/PI marijuana users were as likely as White users to have a marijuana use disorder. In both AA and NH/PI groups, younger age (especially 12-17 years) and a greater frequency of marijuana use ( $\geqslant 52$ days in a year) were robust correlates of marijuana use disorder.

\section{Use of alcohol or drug abuse treatment services}

\section{Surveys of the general populations}

Comparisons of national survey data between the 1991-1992 National Longitudinal Alcohol Epidemiologic Survey and the 2001-2002 NESARC showed some declines in the prevalence of any lifetime treatment service use (addiction or mental health programs, non-specialty medical services, mutual aid, human services, or other service) for alcohol problems among adults with a lifetime alcohol use disorder between the years 1991-1992 and 2001-2002 (Whites $20.3 \%$ v. $14.0 \%$, Blacks $22.2 \%$ v. $17.1 \%$, Hispanics $19.6 \%$ v. $16.2 \%$ respectively) but results are not available for AAs and NHs/PIs (Chartier \& Caetano, 2011).

Findings from the 2001-2002 NESARC indicated that fewer AAs/NHs/PIs with a lifetime alcohol use disorder than other racial/ethnic groups with an alcohol use disorder had ever received any treatment or service for alcohol problems in their lifetime (Cohen et al. 2007) (see Table 4).

In a recent report that analyzed the combined 2003-2011 NSDUH data (SAMHSA, 2013), 4.9\% of pooled AAs/NHs/PIs aged $\geqslant 12$ years were estimated to need treatment for alcohol $(3.9 \%)$ or drug $(1.4 \%)$ abuse (i.e. having a DSM-IV alcohol or drug use disorder or receiving treatment for alcohol or drug use at an addiction or mental health service setting in the past year). Among AAs/NHs/PIs aged 18-25, males and those without health insurance had a higher proportion of needing alcohol or drug abuse treatment services than adolescents and adults older than 25. Among AAs/NHs/PIs with a need for alcohol or abuse treatment services in the past year, 5.3\% received alcohol or abuse treatment at a specialty setting (addiction or mental health programs) in the past year. In addition, more AAs/NHs/PIs with a need for illicit drug abuse treatment received specialty treatment (10.9\%) than AAs/NHs/PIs with a need for alcohol abuse treatment received specialty treatment (3.7\%) (SAMHSA, 2013). These data indicate substantial unmet needs for SUD treatment. 
Table 3. Alcohol and drug use disorders among Asian Americans (AAs) and Native Hawaiians/Pacific Islanders (NHs/PIs)

\begin{tabular}{|c|c|c|c|c|}
\hline Authors/citation & Dataset, Age & Sample size & Substance & Selected findings \\
\hline $\begin{array}{l}\text { Compton et al. } \\
2007\end{array}$ & $\begin{array}{l}\text { National sample } \\
\text { NESARC } \\
\geqslant 18 \text { years }\end{array}$ & 43093 & Drugs & $\begin{array}{l}\text { Lifetime drug use disorder } \\
\text { AA/NH/PI } 3.8 \% \text {, White } 11.3 \% \text {, Black } 8.7 \% \text {, Hispanic } 7.2 \% \text {, Native American } 18.4 \%\end{array}$ \\
\hline Hasin et al. 2007 & $\begin{array}{l}\text { National sample } \\
\text { NESARC } \\
\geqslant 18 \text { years }\end{array}$ & 43093 & Alcohol & $\begin{array}{l}\text { Lifetime alcohol use disorder } \\
\text { AA/NH/PI 11.6\%, White 34.1\%, Black 20.6\%, Hispanic 21.0\%, Native American } 43.0 \%\end{array}$ \\
\hline Wu et al. 2011 & $\begin{array}{l}\text { National sample } \\
\text { NSDUH } \\
\text { 12-17 years }\end{array}$ & 72561 & Alcohol or drugs & $\begin{array}{l}\text { Past-year alcohol or drug use disorder } \\
\text { AA/NH/PI: 3.5\% (alcohol 2.1\%, any drug } 2.0 \% \text { ) } \\
\text { White: } 9.0 \% \text { (alcohol } 6.3 \% \text {, any drug } 5.0 \% \\
\text { Black: } 5.0 \% \text { (alcohol } 2.3 \% \text {, any drug } 3.7 \% \text { ) } \\
\text { Hispanic: } 7.7 \% \text { (alcohol 5.5\%, any drug } 4.4 \% \text { ) } \\
\text { Native American: } 15.0 \% \text { (alcohol } 9.4 \% \text {, any drug } 8.7 \% \text { ) } \\
\text { Mixed-race: } 9.2 \% \text { (alcohol } 6.3 \% \text {, any drug } 6.4 \% \text { ) }\end{array}$ \\
\hline Wu et al. 2012 & $\begin{array}{l}\text { National sample } \\
\text { NSDUH } \\
\geqslant 18 \text { years }\end{array}$ & $\begin{array}{l}113672 \\
\text { users of emergency } \\
\text { department }\end{array}$ & Alcohol or drugs & $\begin{array}{l}\text { Conditional past-year alcohol or drug use disorder in alcohol or drug users who used } \\
\text { emergency-department care in the past year } \\
\text { AA/NH/PI: } 11.7 \% \text { (alcohol } 10.3 \% \text {, any drug } 13.6 \% \text { ) } \\
\text { White: } 15.2 \% \text { (alcohol } 12.5 \% \text {, any drug } 23.3 \% \\
\text { Black: } 18.0 \% \text { (alcohol } 14.4 \% \text {, any drug } 25.7 \% \text { ) } \\
\text { Hispanic: } 17.2 \% \text { (alcohol } 14.6 \% \text {, any drug } 23.4 \% \text { ) } \\
\text { Native American: } 28.7 \% \text { (alcohol } 27.0 \% \text {, any drug } 24.5 \% \text { ) } \\
\text { Mixed-race: } 23.6 \% \text { (alcohol } 21.4 \% \text {, any drug } 22.2 \% \text { ) }\end{array}$ \\
\hline Wu et al. 2014 & $\begin{array}{l}\text { National sample } \\
\text { NSDUH } \\
\geqslant 12 \text { years }\end{array}$ & 394400 & Marijuana & $\begin{array}{l}\text { Past-year marijuana use disorder (conditional prevalence of marijuana use disorders in marijuana users) } \\
\text { AA: } 0.8 \%(17.0 \% \text { in marijuana users) } \\
\text { NH/PI: } 1.3 \%(12.6 \% \text { in marijuana users) } \\
\text { White: } 1.5 \%(13.7 \% \text { in marijuana users) } \\
\text { Black: } 2.5 \%(19.1 \% \text { in marijuana users) } \\
\text { Hispanic: } 1.8 \% \text { (19.6\% in marijuana users) } \\
\text { Native American: } 3.7 \% \text { ( } 24.6 \% \text { in marijuana users) } \\
\text { Mixed-race: } 2.8 \% \text { (15.8\% in marijuana users) }\end{array}$ \\
\hline
\end{tabular}

NESARC, National Epidemiologic Survey on Alcohol and Related Conditions; NSDUH, National Survey on Drug Use and Health. 
Table 4. Substance abuse service use and co-morbidity among Asian Americans (AAs) and Native Hawaiians/Pacific Islanders (NHs/PIs)

\begin{tabular}{|c|c|c|c|c|}
\hline Authors/citation & Dataset, Age & Sample size & Substance & Selected findings \\
\hline Wu et al. 2003 & $\begin{array}{l}\text { National sample } \\
\text { NHSDA } \\
\geqslant 18 \text { years }\end{array}$ & 16661 & Alcohol or drug & $\begin{array}{l}\text { Adjusted OR }(95 \% \mathrm{CI}) \text { of substance abuse service use in adults with alcohol or drug use problems } \\
\text { AA/NH/PI } v \text {. White } 0.28(0.11-0.70) \text {, Black } v \text {. White } 0.73(0.33-1.59) \text {, Hispanic } v \text {. } \\
\text { White } 0.59(0.27-1.30) \text {, Native American } v \text {. White } 4.74(1.88-11.98)\end{array}$ \\
\hline Cohen et al. 2007 & $\begin{array}{l}\text { National sample } \\
\text { NESARC } \\
\geqslant 18 \text { years }\end{array}$ & 1716 & Alcohol & $\begin{array}{l}\text { Any lifetime use of alcohol services in adults with a lifetime alcohol use disorder } \\
\text { AA/NH/PI } 9.0 \% \text {, White } 14.0 \% \text {, Black } 17.1 \% \text {, Hispanic } 16.1 \% \text {, Native American } 22.9 \%\end{array}$ \\
\hline Cummings et al. 2011 & $\begin{array}{l}\text { National sample } \\
\text { NSDUH } \\
12-17 \text { years }\end{array}$ & 12634 & Alcohol or drug & $\begin{array}{l}\text { Any past-year use of alcohol or drug services in adolescents with a past-year alcohol or drug use disorder } \\
\text { AA } 11.0 \% \text {, White } 11.9 \% \text {, Black } 8.4 \% \text {, Hispanic } 9.8 \% \text {, Native American } 18.2 \% \text {, Mixed-race } 15.6 \%\end{array}$ \\
\hline Mericle et al. 2012 & $\begin{array}{l}\text { National sample } \\
\text { CPES } \\
\geqslant 18 \text { years }\end{array}$ & 19729 & Alcohol or drug & $\begin{array}{l}\text { Lifetime disorder in adults with a lifetime alcohol use disorder } \\
\text { Depressive disorder: AA } 41.6 \% \text {, White } 37.6 \% \text {, Black } 27.5 \% \text {, Hispanic } 29.7 \% \\
\text { Anxiety disorders in adults with a lifetime alcohol use disorder: AA } 34.4 \% \text {, White } 41.4 \% \text {, } \\
\text { Black } 36.1 \% \text {, Hispanic } 31.6 \% \\
\text { Lifetime disorder in adults with a lifetime drug use disorder } \\
\text { Depressive disorder: AA } 47.5 \% \text {, White } 43.9 \% \text {, Black } 33.4 \% \text {, Hispanic } 29.3 \% \\
\text { Anxiety disorders in adults with a lifetime alcohol use disorder: AA } 41.1 \% \text {, White } 47.3 \% \text {, } \\
\text { Black } 42.5 \% \text {, Hispanic } 35.2 \%\end{array}$ \\
\hline
\end{tabular}

CPES, Collaborative Psychiatric Epidemiology Surveys; NESARC, National Epidemiologic Survey on Alcohol and Related Conditions; NHSDA, National Household Survey on Drug Use; NSDUH, National Survey on Drug Use and Health; OR, odds ratio; CI, confidence interval.

Any use of treatment services included use of treatment or service at specialty (addiction or mental health) programs, non-specialty medical services, mutual aid, non-specialty human services, or other settings. 
Available but limited national survey data for adolescents suggest few racial/ethnic differences in the prevalence of any substance abuse treatment use among adolescents. Among adolescents aged 12-17 with a past-year alcohol or drug use disorder, Cummings et al. (2011) found that more Native Americans than AAs used any substance abuse treatment in the past year. Mixed race individuals fell between these two groups (see Table 4).

\section{Clinical samples}

Treatment use data from the New York statewide substance abuse treatment and discharge database showed that AAs represented a small proportion $(<1 \%)$ of the patients in the sample (Yu \& Warner, 2013; Yu et al. 2014). About $82 \%$ of AA patients were male; the mean age of AAs was 34.6 years, which did not differ significantly from Whites, Blacks and Hispanics. Close to $64 \%$ of AA patients reported English as their primary language. More AAs had only one treatment episode $(71 \%)$ in the database than Whites $(65 \%)$, Blacks $(56 \%)$ and Hispanics (53\%). The data suggested that AAs (43\%) may be more likely than Whites (31\%), Blacks (39\%) and Hispanics (37\%) to enter treatment through the criminal justice system (Yu et al. 2014).

The Treatment Episode Data Set (TEDS), which collects demographic and substance abuse characteristics of admissions to treatment for abuse of alcohol and/ or drugs in facilities that report to individual state administrative data systems, includes substance abuse treatment admission data for pooled AAs/NHs/PIs. AAs/NHs/PIs accounted for $1 \%$ of all TEDS admissions, and alcohol was the primary substance involved in $46 \%$ of male $\mathrm{AA} / \mathrm{NH} / \mathrm{PI}$ admissions and $38 \%$ of female AA/NH/PI admissions. Among male AAs/NHs/ PIs, marijuana (21\%) and amphetamines/methamphetamines $(17 \%)$ were more frequently identified than opiates (9\%) and cocaine (5\%). Among female AAs/ NHs/PIs, amphetamines/methamphetamines (23\%) and marijuana (19\%) were more commonly identified than opiates (13\%) and cocaine (5\%) (SAMHSA, 2012).

Compared with patterns of substance use in the overall AA/NH/PI admissions, illicit drugs accounted for a high proportion of treatment admissions among young AAs/NHs/PIs (ages 18-25 years) in TEDS. Among these young adults, alcohol was the primary substance involved in $34 \%$ of male admissions and $26 \%$ of female admissions (SAMHSA, 2010b). Methamphetamines and marijuana were the two primary drug classes involved in treatment admissions for AAs/NHs/PIs aged 18-25. The most common source of referrals for young $\mathrm{AA} / \mathrm{NH} / \mathrm{PI}$ admissions was the criminal justice system (male 65\%, female 44\%), followed by self-referral (male 19\%, female 26\%) and other community organizations (male $7 \%$, female $17 \%)$. Other medical settings accounted for a very low proportion $(<7 \%)$ of referrals for substance abuse treatment admissions (SAMHSA, 2010b).

Collectively, the treatment use data from pooled AAs/NHs/PIs in TEDS suggest high proportions of illicit drug-related admissions among young adults, greater methamphetamine use problems among females, and greater marijuana use problems among males. AAs/NHs/PIs with substance abuse problems may be unlikely to be identified or referred to substance abuse treatment by health-care providers.

SUD prevalences among AAs, NHs/PIs and mixed-race people aged $\leqslant 90$ years $(n=4572)$ were examined by $\mathrm{Wu}$ et al. (2013a) in a behavioral health database. DSM-IV diagnoses among patients from 11 hospitals and mental health facilities were systematically captured by an Electronic Health Record system. Overall, $11.3 \%$ of AAs aged $\geqslant 12$ years had an SUD (3.7\% having at least two SUDs). Adolescents aged 12-17 (10.1\%) had a higher prevalence of cannabis diagnosis than adults aged $35-49$ (10.1\% v. 2.1\%). In the adjusted analysis, AA adolescents and young adults (18-34 years) were two to three times more likely than older adults ( $\geqslant 50$ years) to have an SUD. Overall, $20.1 \%$ of $\mathrm{NHs} / \mathrm{PIs}$ aged $\geqslant 12$ years had an SUD (6.5\% having at least two SUDs). Adolescents and young adults aged 18-34 had higher prevalences of cannabis diagnosis than NHs/PIs aged $\geqslant 35$ years, whereas alcohol, cocaine and polysubstance diagnoses were more prevalent among adults than adolescents. Compared with AAs, NHs/PIs had higher odds of having an SUD (aOR 2.62, 95\% CI 2.00-3.42) and multiple SUDs (aOR 2.41, 95\% CI 1.55-3.76), even after the analysis was adjusted for age at first treatment, sex, treatment setting, length in psychiatric treatment, and the number of other co-morbid diagnoses. Greater length of psychiatric treatment among NHs/ PIs was positively associated with having at least two SUDs. In both groups, males were more likely than females to have an SUD or at least two SUDs (Wu et al. 2013a). In summary, treatment-seeking $\mathrm{NH} / \mathrm{PIs}$ showed a higher SUD prevalence than treatment-seeking AAs.

\section{Psychiatric co-morbidities}

Psychiatric co-morbidities can intensify clinical courses and affect treatment outcomes of individuals with an SUD (Najt et al. 2011). However, there are very limited co-morbidity data available for AAs and NHs/PIs. Of the pooled AAs/NHs/PIs $(n=1332)$ in the 2001-2002 NESARC, $7.4 \%$ had a past-year mood disorder (similar to Whites, 9.4\%), $6.9 \%$ had a past-year anxiety disorder 
(lower than Whites, $11.7 \%$ ), and $10.1 \%$ had a personality disorder (lower than Whites, 14.6\%) (Huang et al. 2006). In the NESARC, lifetime alcohol use disorder among pooled AAs/NHs/PIs $(n=1332)$ was associated with past-year mood disorder (aOR 3.6, 95\% CI 1.4-8.9), anxiety disorder (aOR 3.6, 95\% CI 1.4-8.9) and personality disorder (aOR 5.0, 95\% CI 2.1-12.0). Because of the small sample size, associations of drug use disorder with other mental disorder are unclear. In another analysis of more recent national samples, past-year drug use among AAs was associated with major depressive episode (aOR 1.85, 95\% CI 1.26-2.71) (Wu et al. 2013b), and past-year marijuana use disorder among AA marijuana users was associated with major depressive episode (aOR 2.11, 95\% CI 1.10-4.05) (Wu et al. 2014).

Using a pooled sample from the Collaborative Psychiatric Epidemiology Surveys (including the NCS-R, National Survey of American Life, and National Latino and Asian American Study), Mericle et al. (2012) compared lifetime co-morbid prevalences of psychiatric disorders among AAs. Co-morbidity was high, as shown in Table 4. AAs with co-morbid SUD and other mental disorders did not differ from Whites, Blacks and Hispanics in the prevalence of prior psychiatric hospitalization (22.8\% in AAs), history of attempted suicide (17.2\% in AAs) and difficulty in paying bills (57.6\% in AAs). However, a higher proportion of AAs with co-morbid SUD and other mental disorders were unemployed compared with Whites (21.1\% v. 2.5\%) (Mericle et al. 2012).

Research on electronic health record data of community residents $(n=4572)$ provided clinical profiles of AAs and NHs/PIs who accessed mental or substance abuse care at one of the 11 hospitals and mental health centers (Wu et al. 2013a). Comparisons between treatment-seeking AAs with an SUD and treatmentseeking AAs without an SUD showed no significant differences in diagnoses for mood $(50.0 \%$ among AAs with an SUD v. 54.7\% among AAs without an SUD), anxiety $(26.0 \%$ v. $31.3 \%)$, adjustment $(14.4 \%$ v. $22.7 \%)$ or personality disorder $(14.4 \%$ v. $8.8 \%)$; schizophrenic or psychotic (13.5\% v. 17.9\%); attention-deficit/hyperactivity disorder, conduct disorder, oppositional defiant disorder and disruptive behavior $(8.7 \%$ v. $7.7 \%)$; or eating $(3.8 \%$ v. $3.4 \%)$, cognitive $(1.9 \% \quad v$. $2.6 \%)$ or impulse-control disorder $(1.0 \%$ v. $1.7 \%)$. Comparisons between treatment-seeking NHs/PIs with an SUD and treatment-seeking NHs/PIs without an SUD indicated significant differences in diagnoses for mood $(73.6 \%$ among $\mathrm{NHs} / \mathrm{PI}$ with an SUD $v$. $64.2 \%$ among NHs/PIs without an SUD), adjustment $(33.6 \%$ v. $43.6 \%)$ and personality disorder $(20.2 \% v$. $10.9 \%)$. Overall, treatment-seeking NHs/PIs had higher prevalences than treatment-seeking AAs of mood disorder $(62.2 \%$ v. 50.2\%); adjustment disorder $(43.5 \%$ v. 22.1\%); attention-deficit/hyperactivity disorder, conduct disorder, oppositional defiant disorder and disruptive behavior (19.9\% v. $11.8 \%)$; and impulsecontrol disorder (12.2\% v. 1.7\%) diagnoses.

\section{Discussion}

AAs and NHs/PIs are frequently reported as a single group in research and health statistics. Findings from pooled AAs/NHs/PIs indicate a low prevalence of alcohol/drug use but analyses that disaggregate NHs/ PIs from AAs reveal a particularly high prevalence of substance use among NHs/PIs in the general population and treatment-seeking samples. Nationally, an estimated $11 \%$ of $\mathrm{NHs} / \mathrm{PIs}$ aged $\geqslant 12$ years had a history of major depressive episodes (7\% among AAs), 33\% used tobacco in the past year (18\% among AAs), $61 \%$ used alcohol in the past year $(52 \%$ among AAs), and $21 \%$ used illicit or non-medical drugs in the past year ( $7 \%$ among AAs) (Wu et al. $2013 b, c)$. Overall, NHs/PIs resemble Whites in drug use prevalence whereas AAs have lower odds than Whites of using drugs (marijuana, opioids, cocaine, hallucinogens, stimulants, sedatives, tranquilizers). Thus, health statistics from pooled AAs/NHs/PIs may underestimate intervention needs for NHs/PIs.

Compared with AAs, NHs/PIs on average are younger, less educated and less likely to be foreignborn; have lower family incomes; and have greater behavioral risk indicators. Such characteristics, including younger age, US-born, less education, low family income, and behavioral risk indicators (depression, delinquency, suicidal behaviors), are all associated with substance use (SAMHSA, 2010a; Lowry et al. 2011; Wu et al. 2013b,c, 2014). These differences may account partly for some differences in substance use prevalences between NHs/PIs and AAs. Available data, although descriptive, demonstrate that NHs/PIs are a vulnerable population that requires research efforts to discern risk and protective factors of substance use and to develop culturally appropriate interventions to address substance use (Edwards et al. 2010). Adolescents and young adults in addition to low-income NHs/PIs should be included as targets of primary and secondary prevention programs $(\mathrm{Wu}$ et al. 2013b,c, 2014). Marijuana use, accounting for about $89 \%$ of all past-year drug use among NHs/PIs, should be the primary focus of drug use intervention (Wu et al. 2014).

Across AA subgroups, alcohol use is comparatively prevalent among Korean, Japanese and Chinese Americans whereas drug use is comparatively prevalent among Korean, Japanese and Vietnamese Americans (SAMHSA, 2010a). Although AAs use 
fewer substances than NHs/PIs, the burden of substance use problems is growing, especially among Chinese Americans, the largest AA subgroup, as their population size rises rapidly. Alcohol, marijuana and methamphetamines account for most treatment admissions and can be the focus of research and intervention for AAs and NHs/PIs (SAMHSA, 2010b, 2012; Wu et al. 2013a).

Nationally, an estimated $4.5 \%$ of adult AAs/NHs/PIs had a past-year alcohol use disorder and $1.4 \%$ had a past-year drug use disorder (Compton et al. 2007; Hasin et al. 2007). Among adolescent AAs/NHs/ PIs (12-17 years), an estimated $2.1 \%$ had a past-year alcohol use disorder and $2.0 \%$ had a past-year drug use disorder ( $\mathrm{Wu}$ et al. 2011). Males, adolescents and young adults are disproportionally affected by SUDs. More recent data estimate that $0.8 \%$ of AAs $(\geqslant 12$ years) and $1.3 \%$ of NHs/PIs nationally have a past-year marijuana use disorder and that AAs have a lower prevalence than the national average prevalence (1.7\%) (Wu et al. 2014). However, research is needed to provide more reliable estimates of alcohol and drug use disorders specifically for AAs and NHs/PIs.

Although reliable estimates of SUD treatment use among AAs and NHs/PIs are lacking, AAs/NHs/PIs as a group considerably underutilize treatment $(\mathrm{Wu}$ et al. 2003), especially treatment for alcohol problems (SAMHSA, 2013). An estimated 91\% of adult AAs/ $\mathrm{NHs} / \mathrm{PIs}$ in a national sample with a lifetime alcohol use disorder had never used an alcohol abuse treatment service (Cohen et al. 2007). Among AAs/NHs/ PIs ( $\geqslant 12$ years) with a need for alcohol abuse treatment in the past year, only $3.7 \%$ received treatment at a specialty (addiction, mental health) setting in the past year; among AAs/NHs/PIs with a need for drug use treatment, just $10.9 \%$ received drug abuse treatment at a specialty setting (SAMHSA, 2013). Because prevalence estimates are defined to include any service use (specialty plus non-specialty settings, non-medical human services, self-help groups) and treatment often requires multiple visits, the gap of unmet needs for treatment is much greater than the estimates. The low prevalence of treatment use is related to a low rate $(2 \%)$ of perceived need for treatment by AAs/ NHs/PIs with substance problems (SAMHSA, 2013).

Additionally, denial, stigma, cost of treatment, lack of knowledge about treatment, language barriers, and cultural attitudes to behavioral health may contribute to low rate of treatment use (Wu \& Ringwalt, 2004; Wu et al. 2007; Smith et al. 2010; Hedden \& Gfroerer, 2011). AAs may experience a particularly high level of unmet need for SUD care because culturerelate beliefs about occurrences of behavioral and mental illnesses ('Karma') and family-related stigma associated with such conditions (e.g. embarrassment, fear of destroying a family reputation) may delay helpseeking and interfere with engagement in treatment (Li \& Seidman, 2010). Cost concerns (lacking insurance coverage), inadequate English language proficiency and a lack of knowledge about SUD treatment options (which are developed from the western culture) may also impede treatment use (Yu et al. 2009). AAs may delay entering into treatment until substance use impairs daily functioning or causes legal consequences. Treatment use data suggest that AAs tend to use SUD treatment through the criminal justice system (SAMHSA, 2010b; Yu et al. 2014). AAs and NHs/ PIs may benefit from targeted educational interventions that increase their knowledge about the symptoms of SUDs, multiple consequences and the availability of treatment services.

Personality disorder $(10.1 \%)$ is the most prevalent DSM-IV disorder among adult AAs/NHs/PIs ( $\geqslant 18$ years) identified by survey data (NESARC), followed by lifetime mood $(7.4 \%)$, anxiety $(6.9 \%)$, alcohol $(4.5 \%)$ and drug use $(1.4 \%)$ disorders (Huang et al. 2006). US-born AAs ( $\geqslant 18$ years) have a higher lifetime prevalence than foreign-born AAs ( $\geqslant 18$ years) of $\operatorname{mood}(16.6 \%$ v. $11.8 \%)$, anxiety $(17.8 \%$ v. $9.1 \%)$, alcohol $(24.5 \%$ v. $7.3 \%)$ and drug use $(8.3 \%$ v. $2.3 \%)$ disorders (Breslau \& Chang, 2006). Half of AAs ( $\geqslant 18$ years) with a lifetime alcohol or drug use disorder in a national sample had a lifetime mood $(41.0 \%)$ or anxiety (35.3\%) disorder (Mericle et al. 2012). Co-morbid patterns among AAs with an SUD are generally similar to those of Whites, Blacks and Hispanics with an SUD but such survey data for NHs/PIs are lacking. Survey findings are generally limited to descriptive lifetime prevalences, which are inadequate for estimating intervention needs for recent disorders specifically related to AAs and $\mathrm{NHs} / \mathrm{PIs}$.

Mood disorder seems to be the most common DSM-IV diagnosis contributing to use of psychiatric care by AAs and NHs/PIs (Wu et al. 2013a). Among AAs and NHs/PIs with or without an SUD (aged $\geqslant 12$ years), the majority of AAs (50-55\%) and NHs/ PIs (64-74\%) had a mood disorder documented in an electronic behavioral health record database $(\mathrm{Wu}$ et al. 2013a). Treatment-seeking data are consistent with survey findings, showing that $\mathrm{NHs} / \mathrm{PI}$ have a higher prevalence of SUDs than AAs and that personality diagnosis is associated with SUD among AAs and NHs/PIs. Moreover, treatment-seeking NHs/PIs have more DSM-IV diagnoses than treatment-seeking AAs (mood disorder; adjustment disorder; attentiondeficit/hyperactivity disorder, conduct disorder, oppositional defiant disorder, and disruptive behavior; impulse-control disorder), suggesting worse mental health profiles among NHs/PIs than AAs (Lowry et al. 2011; Wu et al. 2013a). 


\section{Future directions}

There is a clear need for proper categorization of AAs and NHs/PIs. The pooled AA and NH/PI group is sometimes described as 'Asian' in research reports. Use of 'Asian' to encompass AAs and NHs/PIs may misinform research and policy, and should be avoided.

The small sample size of AAs and NHs/PIs in typical surveys makes it challenging to have an adequate sample for an in-depth analysis of health status. There is a need for policy support to invest funding in expanding the groups' sample sizes in research projects. One approach is to oversample AAs and NHs/PIs in a given national sample to obtain a sufficiently large sample for estimating substance use indicators for subgroups of AAs and NHs/PIs.

AAs and NHs/PIs are diverse in their cultures and ethnic groups. To design effective intervention programs, research is needed to elucidate substance use behaviors and attitudes regarding treatment among specific ethnic groups (Iwamoto et al. 2012). This can be achieved by focusing on a particular geographic region with a high proportion of AAs (or NHs/PIs) to produce in-depth empirical data from an adequate sample while containing research costs. For example, research efforts can start with large subgroups of AAs, such as Chinese, Filipinos, Asian Indians, Vietnamese, Koreans, and Japanese Americans.

Adolescence and young adulthood are the most reliable correlates of recent substance use whereas other demographics are inconsistent correlates. Additionally, behavioral health problems (use of other substances, delinquency, depression) are closely associated with substance use among AAs and NHs/PIs. This emphasizes the need for prevention programs to start early and target all racial/ethnic groups. AAs and NHs/PIs are vastly under-represented in substance use prevention research (Rehuher et al. 2008). There is a need for research to evaluate the effects of universal and secondary prevention programs (school- and family-based programs) for AAs and NHs/PIs.

Cultural factors that may be unique and that function as risk or protective factors for substance use deserve research (Okamoto et al. 2009). Acculturation (changes in an individual's original cultural patterns as a result of continuous or direct contact with individuals of a different culture) is associated with increased odds of SUD and mental disorders whereas factors related to enculturation (the degree to which an individual endorses values indigenous to their native culture, or ethnic identity measures) are associated with decreased odds of having a mental disorder (Redfield et al. 1936; Blanco et al. 2013; Burnett-Zeigler et al. 2013). It is important to study how preservation and promotion of one's own cultural values, such as participating in racial/ethnic activities and behaviors, and developing a sense of cultural pride, belonging and attachment to one's racial/ethnic group and identity, protect against substance use and psychopathology (Kim \& Omizo, 2010; Blanco et al. 2013; Burnett-Zeigler et al. 2013).

Individuals of multiple races (mixed-race) are a rising population (US Census Bureau, 2011). Mixed-race persons have higher odds than whites and AAs for past-year drug use, are similar to NHs/PIs in drug use, and have the highest prevalence of lifetime major depressive episodes (18.2\%) (Wu et al. 2013b,c). Clinically, mixed-race persons and NHs/PIs also have higher prevalences of SUD and other mental disorders than AAs (Wu et al. 2013a). Mixed-race persons on average have lower household income and are younger than the White and AA populations. Research is needed to increase the accuracy of classifying multiple-race status, and for analysis of and reporting about this population. Although little is known about mixed-race persons' risk factors for substance use, research could explore how socio-economic status interacts with acculturation and enculturation factors and contributes to substance use and mental health conditions.

AAs/NHs/PIs with substance problems are unlikely to enter treatment unless treatment is triggered by the involvement with the criminal justice system (SAMHSA, 2010b, 2013; Masson et al. 2013). It seems that only a subset of severe or problematic illicit drug users (e.g. men, young adults, the less educated) use treatment. Individuals with primary alcohol problems and those with internalizing mental disorders (e.g. women, older adults, the educated) may particularly underuse treatment. Besides culturerelated stigma, research needs to explore multiple levels of barriers and facilitators to treatment (e.g. language inefficiency, immigration status, preference for treatment mode, and support by providers, peers and family) (Griner \& Smith, 2006; Fong \& Tsuang, 2007; Yu et al. 2009; Cabral \& Smith, 2011; Masson et al. 2013).

The low usage rate of substance abuse treatment emphasizes the need for incorporating screening of substance use, brief intervention and referral to treatment (SBIRT) programs into primary care to increase early detection and recognition of substance use status, along with timely interventions for substancerelated problems (Moyer, 2013; Pilowsky \& Wu, 2013; Salvalaggio et al. 2013). The Affordable Care Act (ACA) has expanded access to mental and substance abuse services (i.e. essential health benefits) to millions of Americans (Beronio et al. 2013). An estimated eight in 10 (1.6 million) of eligible uninsured 
AAs/NHs/PIs may qualify either for tax credits to purchase coverage in the Health Insurance Marketplace or for Medicaid or the Children's Health Insurance Program (CHIP) (Wendt et al. 2014). Community outreach can help uninsured individuals learn about the new health insurance coverage. There is also a need to increase the number of trained bilingual staff and health-care providers with the language or cultural skills to support outreach and enrollment efforts, or to provide integrated treatment (SAMHSA, 2014).

\section{Conclusions}

AAs and NHs/PIs differ in aspects of culture, language, immigration status, key socio-economic characteristics, substance use and mental health status. Research reports have traditionally combined both into one group, which provides inadequate information for informing health policy. NHs/PIs are a small but growing population that faces significant health disparities in multiple indicators. NHs/PIs have been overlooked and understudied in the substance and mental health literature. As shown in the Healthy People 2010 Final Review report (National Center for Health Statistics, 2012), NHs/PIs and AAs have the least reliable research data available to evaluate their health status. Their growing population size requires research efforts to produce needed substance use and disorder data that can be used to inform interventions and clinical trials and establish evidenced-based therapies for these ethnically and culturally diverse US populations. Such efforts are necessary for informing the Healthy People 2020 health objectives.

\section{Acknowledgments}

Dr L.-T. $\mathrm{Wu}$ is a committee member of the National Institute on Drug Abuse Asian American and Pacific Islander Researchers and Scholars Workgroup (NIDA AAPI Workgroup), Special Populations Office, National Institute on Drug Abuse, Bethesda, MD, USA. We thank the NIDA Special Populations Office and the NIDA AAPI Workgroup. We thank P. Hoffmann of the Duke Clinical Research Institute for assistance with manuscript preparation.

This work was made possible by research support from the US NIDA and the National Institute on Minority Health and Health Disparities of the National Institutes of Health (R01MD007658, HHSN271200900499P, API-AS.NET2013-01, API-AS. NET2013-02, R01DA019623, R01DA019901 and R33DA027503 to L.-T. Wu), the JP Gibbons Professorship, and the Duke University Department of Psychiatry and Behavioral Sciences.

\section{Declaration of Interest}

None.

\section{References}

Beronio K, Po R, Skopec L, Glied S (2013). Affordable Care Act Expands Mental Health and Substance Use Disorder Benefits and Federal Parity Protections for 62 Million Americans. U.S. Department of Health and Human Services: Washington, DC.

Blanco C, Morcillo C, Alegría M, Dedios MC, Fernández-Navarro P, Regincos R, Wang S (2013). Acculturation and drug use disorders among Hispanics in the U.S. Journal of Psychiatric Research 47, 226-232.

Breslau J, Chang DF (2006). Psychiatric disorders among foreign-born and US-born Asian-Americans in a US national survey. Social Psychiatry and Psychiatric Epidemiology 41, 943-950.

Breslau J, Kendler KS, Su M, Gaxiola-Aguilar S, Kessler RC (2005). Lifetime risk and persistence of psychiatric disorders across ethnic groups in the United States. Psychological Medicine 35, 317-327.

Burnett-Zeigler I, Bohnert KM, Ilgen MA (2013). Ethnic identity, acculturation and the prevalence of lifetime psychiatric disorders among black, Hispanic, and Asian adults in the U.S. Journal of Psychiatric Research 47, 56-63.

Cabral RR, Smith TB (2011). Racial/ethnic matching of clients and therapists in mental health services: a meta-analytic review of preferences, perceptions, and outcomes. Journal of Counseling Psychology 58, 537-554.

Chartier KG, Caetano R (2011). Trends in alcohol services utilization from 1991-1992 to 2001-2002: ethnic group differences in the U.S. population. Alcoholism: Clinical and Experimental Research 35, 1485-1497.

Cohen E, Feinn R, Arias A, Kranzler HR (2007). Alcohol treatment utilization: findings from the National Epidemiologic Survey on Alcohol and Related Conditions. Drug and Alcohol Dependence 86, 214-221.

Compton WM, Thomas YF, Stinson FS, Grant BF (2007). Prevalence, correlates, disability, and comorbidity of DSM-IV drug abuse and dependence in the United States: results from the National Epidemiologic Survey on Alcohol and Related Conditions. Archives of General Psychiatry 64, 566-576.

Cummings JR, Wen H, Druss BG (2011). Racial/ethnic differences in treatment for substance use disorders among U.S. adolescents. Journal of the American Academy of Child and Adolescent Psychiatry 50, 1265-1267.

DeNavas-Walt C, Proctor BD, Smith JC (2012). Income, Poverty, and Health Insurance Coverage in the United States: 2011. US Census Bureau, Current Population Reports, P60-243. US Government Printing Office: Washington, DC

Edwards C, Giroux D, Okamoto SK (2010). A review of the literature on Native Hawaiian youth and drug use: implications for research and practice. Journal of Ethnicity in Substance Abuse 9, 153-172.

Fong TW, Tsuang J (2007). Asian-Americans, addictions, and barriers to treatment. Psychiatry 4, 51-59. 
Griner D, Smith TB (2006). Culturally adapted mental health intervention: a meta-analytic review. Psychotherapy 43, 531-548.

Hasin DS, Stinson FS, Ogburn E, Grant BF (2007). Prevalence, correlates, disability, and comorbidity of DSM-IV alcohol abuse and dependence in the United States: results from the National Epidemiologic Survey on Alcohol and Related Conditions. Archives of General Psychiatry 64, 830-842.

Hedden SL, Gfroerer JC (2011). Correlates of perceiving a need for treatment among adults with substance use disorder: results from a national survey. Addictive Behaviors 36, 1213-1222.

HHS (2013). Asian American/Pacific Islander Profile. US Department of Health and Human Services, Office of Minority Health (http://minorityhealth.hhs.gov/templates/ browse.aspx?lvl=3\&lvlid=29). Accessed 10 January 2014.

Huang B, Grant BF, Dawson DA, Stinson FS, Chou SP, Saha TD, Goldstein RB, Smith SM, Ruan WJ, Pickering RP (2006). Race-ethnicity and the prevalence and co-occurrence of Diagnostic and Statistical Manual of Mental Disorders, Fourth Edition, alcohol and drug use disorders and Axis I and II disorders: United States, 2001 to 2002. Comprehensive Psychiatry 47, 252-257.

Iwamoto D, Takamatsu S, Castellanos J (2012). Binge drinking and alcohol-related problems among U.S.-born Asian Americans. Cultural Diversity and Ethnic Minority Psychology 18, 219-227.

Kim BS, Omizo MM (2010). Behavioral enculturation and acculturation, psychological functioning, and help-seeking attitudes among Asian American adolescents.

Asian American Journal of Psychology 1, 175-185.

Kim J, McCarthy WJ (2006). School-level contextual influences on smoking and drinking among Asian and Pacific Islander adolescents. Drug and Alcohol Dependence 84, 56-68.

Li H, Seidman L (2010). Engaging Asian American youth and their families in quality mental health services. Asian Journal of Psychiatry 3, 169-172.

Lowry R, Eaton DK, Brener ND, Kann L (2011). Prevalence of health-risk behaviors among Asian American and Pacific Islander high school students in the U.S., 2001-2007. Public Health Reports 126, 39-49.

Masson CL, Shopshire MS, Sen S, Hoffman KA, Hengl NS, Bartolome J, McCarty D, Sorensen JL, Iguchi MY (2013). Possible barriers to enrollment in substance abuse treatment among a diverse sample of Asian Americans and Pacific Islanders: opinions of treatment clients. Journal of Substance Abuse Treatment 44, 309-315.

Mericle AA, Ta Park VM, Holck P, Arria AM (2012). Prevalence, patterns, and correlates of co-occurring substance use and mental disorders in the United States: variations by race/ethnicity. Comprehensive Psychiatry 53, 657-665.

Moyer VA, Preventive Services Task Force (2013). Screening and behavioral counseling interventions in primary care to reduce alcohol misuse: U.S. preventive services task force recommendation statement. Annals of Internal Medicine 159, 210-218.
Najt P, Fusar-Poly P, Brambilla P (2011). Co-occurring mental and substance abuse disorders: a review on the potential predictors and clinical outcomes. Psychiatry Research 186, 159-164.

National Center for Health Statistics (2012). Healthy People 2010 Final Review. National Center for Health Statistics: Hyattsville, MD.

Okamoto SK, Helm S, Po'a-Kekuawela K, Chin CI, Nebre LR (2009). Community risk and resiliency factors related to drug use of rural Native Hawaiian youth: an exploratory study. Journal of Ethnicity in Substance Abuse 8, 163-177.

Pilowsky DJ, Wu LT (2013). Screening instruments for substance use and brief interventions targeting adolescents in primary care: a literature review. Addictive Behaviors 38, 2146-2153.

Price RK, Risk NK, Wong MM, Klingle RS (2002). Substance use and abuse by Asian Americans and Pacific Islanders: preliminary results from four national epidemiologic studies. Public Health Reports 117 (Suppl. 1), S39-S50.

Redfield R, Linton R, Herskovits MJ (1936). Memorandum for the study of acculturation. American Anthropologist 38, 149-152.

Rehuher D, Hiramatsu T, Helm S (2008). Evidence-based youth drug prevention: a critique with implications for practice-based contextually relevant prevention in Hawai' $i$. Hawai'i Journal of Public Health 1, 52-61.

Salvalaggio G, Dong K, Vandenberghe C, Kirkland S, Mramor K, Brown T, Taylor M, McKim R, Cummings GG, Wild TC (2013). Enhancing screening, brief intervention, and referral to treatment among socioeconomically disadvantaged patients: study protocol for a knowledge exchange intervention involving patients and physicians. BMC Health Services Research 13, 108.

SAMHSA (2010a). The NSDUH Report: Substance Use Among Asian Adults. Substance Abuse and Mental Health Services Administration, Office of Applied Studies: Rockville, MD.

SAMHSA (2010b). The TEDS Report: Gender Differences Among Asian and Pacific Islander Treatment Admissions Aged 18-25. Substance Abuse and Mental Health Services Administration, Office of Applied Studies: Rockville, MD.

SAMHSA (2011). The NSDUH Report: Substance Use Among Asian Adolescents. Substance Abuse and Mental Health Services Administration, Center for Behavioral Health Statistics and Quality: Rockville, MD.

SAMHSA (2012). Treatment Episode Data Set (TEDS): 2000-2010. National Admissions to Substance Abuse Treatment Services. DASIS Series S-61, HHS Publication No. (SMA) 12-4701. Substance Abuse and Mental Health Services Administration, Center for Behavioral Health Statistics and Quality: Rockville, MD.

SAMHSA (2013). The NSDUH Report: Need for and Receipt of Substance Use Treatment among Asian Americans and Pacific Islanders. Substance Abuse and Mental Health Services Administration, Center for Behavioral Health Statistics and Quality: Rockville, MD.

SAMHSA (2014). Strategies for Behavioral Health Organizations to Promote New Health Insurance Opportunities in Asian American, Native Hawaiian, and Pacific Islander 
Communities. HHS Publication No. (SMA) 14-4818. Substance Abuse and Mental Health Services Administration: Rockville, MD.

Smith SM, Dawson DA, Goldstein RB, Grant BF (2010). Examining perceived alcoholism stigma effect on racial-ethnic disparities in treatment and quality of life among alcoholics. Journal of Studies on Alcohol and Drugs 71, 231-236.

US Census Bureau (2011). Overview of Race and Hispanic Origin: 2010. Economics and Statistics Administration, US Department of Commerce: Washington, DC.

US Census Bureau (2012a). The Asian Population: 2010. Economics and Statistics Administration, US Department of Commerce: Washington, DC.

US Census Bureau (2012b). The Native Hawaiian and Other Pacific Islander Population: 2010. Economics and Statistics Administration, US Department of Commerce: Washington, DC.

US Census Bureau (2013). Asian/Pacific American Heritage Month: May. Economics and Statistics Administration, US Department of Commerce: Washington, DC (www. census.gov/newsroom/releases/pdf/cb13ff-09_asian.pdf). Accessed 10 January 2014.

Wendt M, Wilson-Frederick S, Wu S, Gee ER (2014). Eligible uninsured Asian Americans, Native Hawaiians, and Pacific Islanders: 8 in 10 could receive health insurance marketplace tax credits, MEDICAID or CHIP. US Department of Health and Human Services: Washington, DC.

Wong MM, Klingle RS, Price RK (2004). Alcohol, tobacco, and other drug use among Asian American and Pacific Islander adolescents in California and Hawaii. Addictive Behaviors 29, 127-141.

Wu LT, Blazer DG, Gersing KR, Burchett B, Swartz MS, Mannelli P; NIDA AAPI Workgroup (2013a). Comorbid substance use disorders with other Axis I and II mental disorders among treatment-seeking Asian Americans, Native Hawaiians/Pacific Islanders, and mixed-race people. Journal of Psychiatric Research 47, 1940-1948.

Wu LT, Blazer DG, Swartz MS, Burchett B, Brady KT; NIDA AAPI Workgroup (2013b). Illicit and nonmedical drug use among Asian Americans, Native Hawaiians/Pacific Islanders, and mixed-race individuals. Drug and Alcohol Dependence 133, 360-367.

Wu LT, Brady KT, Mannelli P, Killeen TK; NIDA AAPI Workgroup (2014). Cannabis use disorders are comparatively prevalent among nonwhite racial/ethnic groups and adolescents: a national study. Journal of Psychiatric Research 50, 26-35.

Wu LT, Pilowsky DJ, Schlenger WE, Hasin D (2007). Alcohol use disorders and the use of treatment services among college-aged young adults. Psychiatric Services 58, 192-200.

Wu LT, Ringwalt CL (2004). Alcohol dependence and use of treatment services among women in the community. American Journal of Psychiatry 161, 1790-1797.

Wu LT, Ringwalt CL, Williams C (2003). Use of substance abuse treatment services by persons with mental health and substance use problems. Psychiatric Services 54, 363-369.

Wu LT, Swartz MS, Burchett B, Workgroup NA, Blazer DG (2013c). Tobacco use among Asian Americans, Native Hawaiians/Pacific Islanders, and mixed-race individuals: 2002-2010. Drug and Alcohol Dependence 132, 87-94.

Wu LT, Swartz MS, Wu Z, Mannelli P, Yang C, Blazer DG (2012). Alcohol and drug use disorders among adults in emergency department settings in the United States. Annals of Emergency Medicine 60, 172-180.e5.

Wu LT, Woody GE, Yang C, Pan JJ, Blazer DG (2011). Racial/ ethnic variations in substance-related disorders among adolescents in the United States. Archives of General Psychiatry 68, 1176-1185.

Yu J, Clark LP, Chandra L, Dias A, Lai TF (2009). Reducing cultural barriers to substance abuse treatment among Asian Americans: a case study in New York City. Journal of Substance Abuse Treatment 37, 398-406.

Yu J, Warner LA (2013). Substance abuse treatment readmission patterns of Asian Americans: comparisons with other ethnic groups. American Journal of Drug and Alcohol Abuse 39, 23-27.

Yu J, Warner LA, Haverly K, Lambert-Wacey D (2014). Service utilization of Asians and other racial-ethnic groups: comparisons in a state substance abuse treatment system. Substance Use and Misuse 49, 205-210. 